\title{
Erectile dysfunction in patients with liver disease relat- ed to chronic hepatitis B
}

\author{
Min Kim, Seul Young Kim, Woo Sun Rou, Se Woong Hwang, and Byung Seok Lee \\ Department of Internal Medicine, Chungnam National University College of Medicine, Daejeon, Korea
}

Background/Aims: Despite sexual function making an important contribution to the quality of life, data on erectile function are relatively scant in patients with chronic liver disease. We evaluated the prevalence of and risk factors for erectile dysfunction (ED) in patients with liver disease related to hepatitis $B$, especially among those with chronic hepatitis B (CHB) or early-stage cirrhosis.

Methods: In total, 69 patients ( 35 with CHB and 34 with hepatitis-B-related liver cirrhosis [HBV-LC]) aged 40-59 years were analyzed. Child-Pugh classes of A and B were present in $30(88.2 \%)$ and $4(11.8 \%)$ of the patients with HBV-LC, respectively. The erectile function of the patients was evaluated using the Korean version of IIEF- 5 .

Results: The prevalence of any ED was $24.6 \%$ for all patients, and $8.6 \%$ and $41.2 \%$ for those with CHB and HBV-LC, respectively $(P=0.002)$. While there was only one (2.9\%) CHB patient for each stage of $E D$, mild, moderate, and severe $E D$ stages were seen in three (8.8\%), one (2.9\%), and ten (29.4\%) of the HBV-LC patients, respectively. Multiple regression analysis identified the type of liver disease $(P=0.010)$, hypertension $(P=0.022)$, score on the Beck Depression Inventory $(P$ $=0.044)$, and the serum albumin level $(P=0.014)$ as significant independent factors for the presence of ED.

Conclusions: The prevalence of ED was significantly higher in patients with early-stage HBV-LC than in those with CHB. Therefore, screening male patients with early viral cirrhosis for ED and providing appropriate support are needed, especially when the cirrhosis is accompanied by hypertension, depression, or a depressed level of serum albumin. (Clin Mol Hepatol 2015;21:352-357)

Keywords: Liver disease; Chronic hepatitis B; Liver cirrhosis; Erectile dysfunction

\section{INTRODUCTION}

Erectile dysfunction (ED) is defined as the inability to obtain or maintain a penile erection sufficient for achieving satisfactory sexual activity. ${ }^{1}$ ED has become an increasing issue because sexual functioning is an important component of good quality of life. Its prevalence is estimated to be about $35 \%$ in Korea and increased with age. ${ }^{2,3} \mathrm{ED}$ can be organic, psychogenic and mixed. It used to be thought that most cases of ED were psychological in origin, but now it is known that organic causes are responsible for at least $80 \%$ of ED cases. ${ }^{4}$ The organic factors include various medical diseases such as cardiovascular disease, genitourinary disease, diabetes mellitus and hypertension.

In respect to sexual dysfunction and liver disease, most studies have been conducted in patients with decompensated cirrhosis, those waiting liver transplants, or those with alcoholic liver cirrho-

\section{Abbreviations:}

$A L T$, alanine aminotransferase; AST, aspartate aminotransferase; BMI, body mass index; $C H B$, chronic hepatitis $B$; $E D$, erectile dysfunction; $H B V$, hepatitis B virus; IIEF-5, simplified International Index of Erectile Function; HBV-LC, hepatitis B-related liver cirrhosis; K-BDI, Korean version of Beck Depression Inventory

\author{
Corresponding author : Byung Seok Lee \\ Division of Gastroenterology, Department of Internal Medicine, \\ Chungnam National University College of Medicine, 282 Munwha-ro, \\ Jung-gu, Daejeon 35015, Korea \\ Tel: +82-42-280-7125, Fax: +82-42-280-7143 \\ E-mail: gie001@cnuh.co.kr
}


sis. ${ }^{5-7}$ Patients with advanced liver disease often present with clinical features of hypogonadism, such as ED, infertility, decreased libido and testicular atrophy. ${ }^{8}$ In addition, the severity of liver cirrhosis has shown to correlate with the degree of ED. ${ }^{9}$ However, few data are available about erectile function in patients with HBV-related liver disease, especially with chronic hepatitis or early stage of cirrhosis.

The simplified International Index of Erectile Function (IIEF-5) is a reliable and sensitive evaluation instrument for assessing erectile capacity. The Korean version of the IIEF-5 was validated by Ahn et al. ${ }^{2}$

In the present study, we investigated the prevalence and characteristics of ED in patients with HBV-related chronic liver disease using the IIEF-5 questionnaire.

\section{MATERIALS AND METHODS}

\section{Patients}

Middle-aged (40-59 years-old) men with HBV-related liver cirrhosis (HBV-LC) and age-matched patients with chronic hepatitis $\mathrm{B}(\mathrm{CHB})$, who visited the liver unit at the Chungnam National University Hospital from August 2012 to February 2013, were enrolled in this study. The case of LC was defined as a patient having at least one clinical sign of portal hypertension or having ultrasound findings suggestive of cirrhosis of liver. Cirrhotic patients were sub-grouped according to Child-Pugh classification into $A, B$ and $C$. Patients with spinal cord injury, neurologic deficit, a past history of surgery for urological or bowel disease which might affect sexual potency, Child-Pugh class C cirrhosis, or Karnofsky performance status of below $70 \%$ were excluded.

\section{Methods}

We conducted a telephone survey of the patients to assess erectile function, depression, smoking and alcohol consumption. Erectile function of the patients was evaluated by the Korean version of IIEF-5. It consists of 5 items; erection confidence, erection firmness, maintenance ability, maintenance frequency, and satisfaction. Each item is scored on a five-point scale. The respondents were categorized into no (IIEF-5 score $\geq 18$ ), mild (14-17), moderate (10-13) and severe ED $(\leq 9)$. Patients were screened for depression using the Korean version of Beck Depression Inventory $(\mathrm{K}-\mathrm{BDI})$, a widely used 21-item self-rating scale. Scores are inter- preted as follows: $<9$, no depression; 10-15, mild depression; 1623, moderate depression; and $>24$, severe depression. In this study, we simply classified the patients as depressed (K-BDI score $\geq 10$ ) or not. Patients who drank alcohol at least once per week were classified as drinkers. Current smokers were classified as smokers. The response rate was $77.5 \%$; 20 patients did not answer the phone or refused to respond to survey questions. A total of 69 patients were finally included in this study. Two patients who did not answer the K-BDI were excluded from the analysis of depression.

We retrospectively reviewed patient medical records within 2 months prior to the telephone survey and collected the following demographic and clinical data: age; gender; body mass index (BMI); hepatitis B envelop antigen ( $\mathrm{HBeAg}) ;$ HBV DNA; aspartate aminotransferase (AST); alanine aminotransferase (ALT); total bilirubin; serum albumin; prothrombin time; propranolol treatment; antiviral treatment; and comorbidities including hypertension, diabetes mellitus, ascites, and hepatic encephalopathy.

\section{Statistical analyses}

Data are expressed as means \pm SD. A $P$-value $<0.05$ was considered significant. Descriptive statistics were used for frequency analyses. Intergroup comparisons were performed using the $\chi^{2}$ test for qualitative variables, and the independent $t$-test for quantitative variables. Multivariate logistic regression analysis was conducted to assess the independent variables that affected ED. Statistical analyses were carried out using SPSS ver. 18.0 software (SPSS Inc., Chicago, IL, USA).

\section{RESULTS}

The baseline characteristics of the 69 enrolled patients are shown in Table 1. The mean ages of patients with CHB and HBVLC were $49.9 \pm 5.0$ and $50.5 \pm 6.3$ years old, respectively. Of 35 patients with $\mathrm{CHB}, 15$ were in their $40 \mathrm{~s}$ and 20 were in their 50 s. Twelve of 34 patients with HBV-LC were in their 40s and the rest 22 were in their 50s. The Child-Pugh class in patients with HBVLC was A in 30 patients (88.2\%) and B in 4 (11.8\%). No differences were found with regard to BMI, diabetes mellitus, hypertension, alcohol, smoking, depression, antiviral treatment and HBV viral load between patients with CHB and HBV-LC. As expected, HBeAg-positive rate, serum AST, ALT and total bilirubin concentrations were higher while serum albumin level was lower in HBV- 
Table 1. Baseline characteristics of patients with chronic HBV-related liver disease

\begin{tabular}{|c|c|c|c|c|}
\hline & All patients $(n=69)$ & CHB $(n=35)$ & HBV LC $(n=34)$ & $P$-value \\
\hline Age (years) & $50.2 \pm 5.7$ & $49.9 \pm 5.0$ & $50.5 \pm 6.3$ & 0.671 \\
\hline BMI $\left(\mathrm{kg} / \mathrm{m}^{2}\right)$ & $23.9 \pm 3.0$ & $24.3 \pm 2.7$ & $23.4 \pm 3.2$ & 0.232 \\
\hline Diabetes mellitus & $16(23.2)$ & $6(17.1)$ & $10(29.4)$ & 0.227 \\
\hline Hypertension & $19(27.5)$ & $12(34.3)$ & $7(20.6)$ & 0.203 \\
\hline Drinker & $24(34.8)$ & $15(42.9)$ & $9(26.5)$ & 0.153 \\
\hline Smoker & $19(27.5)$ & $10(28.6)$ & $9(26.5)$ & 0.845 \\
\hline Propranolol & $5(7.2)$ & 0 & $5(14.7)$ & 0.025 \\
\hline Antiviral therapy & $55(79.7)$ & 31 (88.6) & $24(70.6)$ & 0.078 \\
\hline HBeAg positive & $28(40.6)$ & $19(54.3)$ & $9(26.5)$ & 0.046 \\
\hline HBV DNA ( $\log _{10}$ copies/mL) & $2.7 \pm 1.5$ & $2.4 \pm 0.9$ & $3.1 \pm 1.9$ & 0.064 \\
\hline Depression (BDI>10) & $11(16.4)$ & $4(11.8)$ & $7(21.2)$ & 0.297 \\
\hline \multicolumn{5}{|l|}{ Child-Pugh classification } \\
\hline A & - & - & $30(88.2)$ & \\
\hline B & - & - & $4(11.8)$ & \\
\hline AST (IU/I) & $28.6 \pm 14.8$ & $22.1 \pm 11.2$ & $35.2 \pm 15.2$ & $<0.001$ \\
\hline $\mathrm{ALT}(\mathrm{IU} / \mathrm{I})$ & $30.4 \pm 18.0$ & $24.9 \pm 16.2$ & $36.1 \pm 18.1$ & 0.008 \\
\hline Total bilirubin (mg/dL) & $1.0 \pm 0.5$ & $0.9 \pm 0.3$ & $1.2 \pm 0.6$ & 0.005 \\
\hline Albumin (g/dL) & $4.3 \pm 0.8$ & $4.4 \pm 0.3$ & $4.2 \pm 0.4$ & 0.009 \\
\hline Prothrombin time (INR) & $1.2 \pm 0.3$ & $1.1 \pm 0.2$ & $1.3 \pm 0.3$ & 0.060 \\
\hline
\end{tabular}

Values are expressed as means \pm SD or $n(\%)$.

BDI, Beck depression inventory; BMI, body mass index; CHB, chronic hepatitis B; HBV, hepatitis B virus; LC, liver cirrhosis; HBeAg, hepatitis B envelop antigen.

Table 2. Prevalence of ED in patients with chronic HBV-related liver disease

\begin{tabular}{|c|c|c|c|c|c|c|}
\hline & Total $(n=69)$ & CHB $(n=35)$ & HBV LC ( $(n=34)$ & $P$-value* & HBV LC-A $(n=30)$ & $P$-value ${ }^{\dagger}$ \\
\hline IIEF-5 score & $18.8 \pm 6.5$ & $21.0 \pm 3.8$ & $16.5 \pm 7.9$ & 0.004 & $17.0 \pm 7.7$ & 0.013 \\
\hline Incidence of ED & $17(24.6)$ & $3(8.6)$ & $14(41.2)$ & 0.002 & $11(36.7)$ & 0.006 \\
\hline ED grade & & & & 0.002 & & 0.010 \\
\hline No & $52(75.4)$ & $32(91.4)$ & $20(58.8)$ & & $19(63.3)$ & \\
\hline Mild & $4(5.8)$ & $1(2.9)$ & $3(8.8)$ & & $2(6.7)$ & \\
\hline Moderate & $2(2.9)$ & $1(2.9)$ & $1(2.9)$ & & $1(3.3)$ & \\
\hline Severe & $11(15.9)$ & $1(2.9)$ & $10(29.4)$ & & $8(26.7)$ & \\
\hline
\end{tabular}

Values are expressed as means \pm SD or $n(\%)$.

ED, erectile dysfunction; CHB, chronic hepatitis B; HBV, hepatitis B virus; HBV LC-A, HBV LC-Child-Pugh grade A; IIEF-5, five-item International Index of Erectile Function; LC, liver cirrhosis.

${ }^{*} P$-values for $\mathrm{CHB}$ versus $\mathrm{HBV} L \mathrm{LC} ;{ }^{\dagger} P$-values for $\mathrm{CHB}$ versus HBV LC-A.

LC patients compared to CHB patients.

The prevalence of any ED was found to be $24.6 \%$ for all patients, and $8.6 \%$ and $41.2 \%$ for CHB and HBV-LC, respectively (Table 2). The mean IIEF-5 score in the ED patients was $8.5 \pm 4.8$. Whereas there was only one $(2.9 \%)$ CHB patient for each stage of $E D$, mild ED was seen in three (8.8\%), moderate ED in one $(2.9 \%)$, and severe ED in ten (29.4\%) in HBV-LC patients. When divided the HBV-LC patients according to the Child-Pugh classification, 2 $(6.7 \%)$ of 30 patients with class A had mild, 1 (3.3\%) had moderate and $8(26.7 \%)$ had severe ED. Of 4 patients with class B, 1 (25\%) had mild and 2 (50\%) had severe ED.

Table 3 shows the clinical and laboratory characteristics of pa- 
Table 3. Risk factors for ED in patients with chronic HBV-related liver disease

\begin{tabular}{|c|c|c|c|}
\hline & $\operatorname{ED}(n=17)$ & No ED $(n=52)$ & $P$-value \\
\hline Liver cirrhosis & $14(82.4)$ & $20(38.5)$ & 0.002 \\
\hline Age (years) & $51.4 \pm 5.9$ & $49.8 \pm 5.6$ & 0.338 \\
\hline BMI $\left(\mathrm{kg} / \mathrm{m}^{2}\right)$ & $24.5 \pm 2.9$ & $23.7 \pm 3.0$ & 0.319 \\
\hline Diabetes mellitus & $7(41.2)$ & $9(17.3)$ & 0.043 \\
\hline Hypertension & $9(52.9)$ & $10(19.2)$ & 0.007 \\
\hline Drinker & $6(35.3)$ & $18(34.6)$ & 0.959 \\
\hline Smoker & $4(23.5)$ & $15(28.8)$ & 0.670 \\
\hline Propranolol & $3(17.6)$ & $2(3.8)$ & 0.092 \\
\hline Antiviral therapy & $14(82.4)$ & $41(78.8)$ & 0.755 \\
\hline Depression (BDI >10) & $5(33.3)$ & $6(11.5)$ & 0.045 \\
\hline AST (IU/I) & $35.6 \pm 18.8$ & $26.3 \pm 12.6$ & 0.070 \\
\hline ALT (IU/I) & $32.2 \pm 18.7$ & $29.8 \pm 17.9$ & 0.630 \\
\hline Total bilirubin (mg/dL) & $1.1 \pm 0.8$ & $1.0 \pm 0.4$ & 0.600 \\
\hline Albumin (g/dL) & $4.1 \pm 0.5$ & $4.4 \pm 0.3$ & 0.020 \\
\hline Prothrombin time (INR) & $1.3 \pm 0.3$ & $1.2 \pm 0.3$ & 0.319 \\
\hline
\end{tabular}

Values are expressed as means \pm SD or $n(\%)$.

$\mathrm{BDI}$, Beck depression inventory; $\mathrm{BMI}$, body mass index; $\mathrm{ED}$, erectile dysfunction; HBV, hepatitis B virus.

Table 4. Multivariable logistic regression for the relative risk of ED

\begin{tabular}{lccc}
\hline & OR & 95\% Cl & P-value \\
\hline Age (years) & 0.974 & $0.832-1.140$ & 0.738 \\
BMI $\left(\mathrm{kg} / \mathrm{m}^{2}\right)$ & 1.305 & $0.942-1.808$ & 0.110 \\
Liver cirrhosis & 39.116 & $2.436-628.030$ & 0.010 \\
Diabetes mellitus & 0.868 & $0.128-5.883$ & 0.885 \\
Hypertension & 30.462 & $1.650-562.424$ & 0.022 \\
Albumin $(\mathrm{g} / \mathrm{dL})$ & 0.037 & $0.003-0.516$ & 0.014 \\
BDI $(>10)$ & 9.737 & $1.066-88.925$ & 0.044 \\
\hline
\end{tabular}

ED, erectile dysfunction; $\mathrm{BDI}$, Beck depression inventory; $\mathrm{BMI}$, body mass index; $\mathrm{Cl}$, confidence interval; $\mathrm{OR}$, odds ratio.

tients with HBV-related liver disease in the presence or absence of ED. The prevalence of LC ( $82.4 \%$ vs. $38.5 \%, P=0.002)$, diabetes mellitus (41.2 vs. $17.3 \%, P=0.043$ ), hypertension (52.9 vs. $19.2 \%$, $P=0.007)$ and depression (33.3\% vs. $11.5 \%, P=0.045)$ was increased in patients with ED than in those without ED. Serum albumin level was $4.1 \pm 0.5 \mathrm{~g} / \mathrm{dL}$ in the ED patients, a value significantly less than that of $4.4 \pm 0.3 \mathrm{~g} / \mathrm{dL}$ in the non-ED patients $(P=0.020)$.

Multiple logistic regression analysis showed that the risk of ED in HBV-LC patients was about 39 times higher than that in $\mathrm{CHB}$ patients ( $\mathrm{OR}=39.12, .95 \% \mathrm{Cl}=2.436-628.030, P=0.010$; Table 4). The odds ratios for $\mathrm{ED}$ were 30.46 (95\% Cl=1.650-562.424, $P=0.022)$ and $9.74(95 \% \mathrm{Cl}=1.066-88.925, P=0.044)$ in patients with hypertension and depression, respectively. High serum albumin levels were found to reduce the risk of $E D(O R=0.04,95 \%$
$\mathrm{Cl}=0.003-0.516, P=0.014)$.

\section{DISCUSSION}

Our data reveal a higher prevalence of ED in HBV-LC patients compared with CHB patients. Surprisingly, this difference remained significant after excluding 4 patients with Child-Pugh class B cirrhosis. In addition, mean IIEF-5 score was significantly lower in patients with Child-Pugh class A cirrhosis than in those with CHB. These results are consistent with the findings of Toda et al. ${ }^{10}$ They reported that the prevalence of moderate and severe ED in patients with early viral cirrhosis, predominantly hepatitis Crelated, is higher than that in those with chronic viral hepatitis 
(60\% vs. $33 \%$ ). On the contrary, Simsek et al. did not show any difference in the prevalence of ED between patients with viral carriers $(51.1 \%)$, chronic hepatitis $(50 \%)$, and cirrhosis $(50 \%)$, respectively. ${ }^{11}$ The most common etiology was HBV infection, however, that study was based on small numbers of cirrhotic patients (only 10 of 81 patients, $12 \%$ ).

In alcoholic cirrhotic men, ED can be associated with a direct toxic effect of ethanol and acetaldehyde on the gonads. ${ }^{12}$ However, the pathophysiology of ED in patients with chronic viral liver disease has not yet been fully determined. In this study, there were significant differences in serum albumin levels between patients with ED and without ED. Furthermore, high levels of serum albumin reduced the risk of $E D$ in multiple regression analyses. A correlation between serum albumin and IIEF score was previously reported by Toda et al..$^{10}$ Decreased production of albumin might influence the ratio of albumin-bound to free testosterone related to sexual desire and sleep-related erection, as well as cause water retention and loss of muscle volume, thereby decreasing physical function of the patients. ${ }^{13}$ Propranolol, which has become widely used to treat portal hypertension, have a negative impact on erectile function. ${ }^{14,15}$ In present study, 5 of 34 HBV LC patients received propranolol. Even after excluding 5 patients receiving propranolol, a higher prevalence of ED in HBV-LC patients compared with CHB patients was observed (37.9\% vs. $8.6 \%, P=0.05)$.

Depression, by reducing sexual desire and physical activity, can cause ED. Chronic infection with HBV is related to several psychiatric disorders including anxiety and depression. ${ }^{16}$ We found a significant association between depression and ED in patients with HBV-related chronic liver disease in this study. Medications, including interferon, for chronic HBV infection, may cause depression as an adverse effect. However, there were no patients treated with interferon in this study. In addition, there was no difference in the prevalence of depression according to the type of antiviral agents.

Hypertension can lead to ED as a consequence of high blood pressure per se or due to antihypertensive treatment. ${ }^{17}$ High blood pressure induces morphological modifications and dysfunction of endothelial cells in the penile vascular bed, contributing to ED. ${ }^{18}$ In the present study, hypertension was shown to highly correlate with ED. We also found that the prevalence of diabetes mellitus, which is one of the most common causes of ED, was higher in patients with than in those without ED. However, it did not reach statistical significance in multiple regression analyses that adjusted for potential confounders.

The limitation in this study is the lack of controls chosen from general population without liver disease. Cho et al. analyzed 827 Korean general population who visited 10 outpatient clinics and reported $33.5 \%$ and $64.4 \%$ prevalence rates of ED for men in their 40 s and 50 s, respectively. ${ }^{3}$ They also described that $64 \%$ of men with chronic diseases, such as hypertension, diabetes, hyperlipidemia, stroke, and myocardial infarction had ED. In our study, the prevalence of ED in patients with $\mathrm{CHB}$ in their $40 \mathrm{~s}$ and $50 \mathrm{~s}$ was only $8.6 \%$. This could be explained by differences in questionnaire response rates (77\% vs. $50 \%$ ), survey methods (telephone survey vs. self-administered questionnaire survey), and types of clinics (primary vs. tertiary) between our study and the study by Cho et al. Other limitations of this study include its small sample size, selection bias and response bias.

In conclusion, the present study showed that the prevalence of ED was significantly increased in patients with early stage of HBV$\mathrm{LC}$ than in those with CHB. Therefore, screening male patients with early viral cirrhosis, especially if accompanied by hypertension, depression, or a depressed level of serum albumin, for ED and providing appropriate support are needed.

\section{Conflicts of Interest}

The authors have no conflicts to disclose.

\section{REFERENCES}

1. Lue TF. Erectile dysfunction. N Engl J Med 2000;342:1802-1813.

2. Ahn TY, Park JK, Lee SW, Hong JH, Park NC, Kim JJ, et al. Prevalence and risk factors for erectile dysfunction in Korean men: results of an epidemiological study. J Sex Med 2007;4:1269-1276.

3. Cho BL, Kim YS, Choi YS, Hong MH, Seo HG, Lee SY, et al. Prevalence and risk factors for erectile dysfunction in primary care: results of a Korean study. Int J Impot Res 2003;15:323-328.

4. Gareri P, Castagna A, Francomano D, Cerminara G, De Fazio P. Erectile dysfunction in the elderly: an old widespread issue with novel treatment perspectives. Int J Endocrinol 2014;2014:878670.

5. Sorrell JH, Brown JR. Sexual functioning in patients with endstage liver disease before and after transplantation. Liver Transpl 2006;12:1473-1477.

6. Huyghe E, Kamar N, Wagner F, Capietto AH, El-Kahwaji L, Muscari F, et al. Erectile dysfunction in end-stage liver disease men. J Sex Med 2009;6:1395-1401.

7. Cornely CM, Schade RR, Van Thiel DH, Gavaler JS. Chronic advanced liver disease and impotence: cause and effect? Hepatology 1984;4:1227-1230.

8. Karagiannis A, Harsoulis F. Gonadal dysfunction in systemic dis- 
eases. Eur J Endocrinol 2005;152:501-513.

9. Wiesner RH, McDiarmid SV, Kamath PS, Edwards EB, Malinchoc M, Kremers WK, et al. MELD and PELD: application of survival models to liver allocation. Liver Transpl 2001;7:567-580.

10. Toda K, Miwa Y, Kuriyama S, Fukushima H, Shiraki M, Murakami N, et al. Erectile dysfunction in patients with chronic viral liver disease: its relevance to protein malnutrition. J Gastroenterol 2005;40:894900.

11. Simsek I, Aslan G, Akarsu M, Koseoglu H, Esen A. Assessment of sexual functions in patients with chronic liver disease. Int J Impot Res 2005;17:343-345.

12. Gluud C, Wantzin P, Eriksen J. No effect of oral testosterone treatment on sexual dysfunction in alcoholic cirrhotic men. Gastroenterology 1988;95:1582-1587.

13. Schiavi RC, Schreiner-Engel P, White D, Mandeli J. The relationship between pituitary-gonadal function and sexual behavior in healthy aging men. Psychosom Med 1991;53:363-374.

14. Smith PJ, Talbert RL. Sexual dysfunction with antihypertensive and antipsychotic agents. Clin Pharm 1986;5:373-384.

15. Fogari $R$, Zoppi A. Effect of antihypertensive agents on quality of life in the elderly. Drugs Aging 2004;21:377-393.

16. Keskin G, Gümüs $A B$, Orgun F. Quality of life, depression, and anxiety among hepatitis B patients. Gastroenterol Nurs 2013;36:346356.

17. Kloner R. Erectile dysfunction and hypertension. Int J Impot Res 2007;19:296-302.

18. Mikhailidis DP, Khan MA, Milionis HJ, Morgan RJ. The treatment of hypertension in patients with erectile dysfunction. Curr Med Res Opin 2000;16( Suppl 1):S31-S36. 